\title{
L'art du Qin. Deux textes d'esthétique musicale chinoise
}

Bruxelles : Institut belge des hautes études chinoises, 1990. 209 p.

[Mélanges chinois et bouddhiques 23]

Juan Martinez

\section{(2) OpenEdition}

\section{Journals}

Édition électronique

URL : http://journals.openedition.org/ethnomusicologie/2454

ISSN : 2235-7688

Éditeur

ADEM - Ateliers d'ethnomusicologie

Édition imprimée

Date de publication : 1 janvier 1992

Pagination : 287-289

ISBN : 978-2-8257-0456-1

ISSN : $1662-372 X$

\section{Référence électronique}

Juan Martinez, "L'art du Qin. Deux textes d'esthétique musicale chinoise », Cahiers d'ethnomusicologie [En ligne], 5 | 1992, mis en ligne le 15 décembre 2011, consulté le 19 avril 2019. URL : http:// journals.openedition.org/ethnomusicologie/2454

Ce document a été généré automatiquement le 19 avril 2019 


\section{L'art du Qin. Deux textes d'esthétique musicale chinoise}

Bruxelles : Institut belge des hautes études chinoises, 1990. 209 p.

[Mélanges chinois et bouddhiques 23]

\section{Juan Martinez}

\section{RÉFÉRENCE}

L'art du Qin. Deux textes d'esthétique musicale chinoise. Traduits et commentés par Georges GOORMAGHTIGH. Bruxelles : Institut belge des hautes études chinoises, 1990. 209 p.

[Mélanges chinois et bouddhiques 23]

1 Les thèses ne font pas forcément de bons livres. Le savoir seul n'ayant par lui-même pas de pouvoir de vérité ou de beauté. Et les résultats d'une recherche méthodique produisent trop souvent un effet de dissuasion à l'égard du lecteur curieux et attentif. Ce truisme ne semble pourtant pas avoir résonné dans les salles de cours des hautes écoles. L'université excelle dans les travaux de laboratoire ; mais en les protégeant de l'extérieur, la vie désespère d'y pénétrer. Ce n'est, disons-le d'emblée, aucunement le cas de l'ouvrage de Georges Goormaghtigh. Chargé d'enseignement à l'Université de Genève, il a entrepris de traduire du chinois deux traités d'esthétique musicale. Il s'est frotté d'une part à « La description poétique du qin » (Qinfu), un texte du troisième siècle de notre ère et, d'autre part, aux «Vingt-quatre saveurs du qin» (Xishan qinkuang), un écrit de Xu Shangying datant du XVII ${ }^{\mathrm{e}}$ siècle. Le qin, une cithare sur table convexe et oblongue, marquée de treize points indiquant les nœuds d'harmoniques, montée de sept cordes de soie, possède une dimension symbolique qui en faisait l'instrument de prédilection des lettrés. Sa partie supérieure, bombée comme le ciel, et sa partie inférieure, plate comme la terre, le désignent comme un véritable microcosme, un instrument « pour harmoniser le Ciel et la Terre ». 
2 Pour l'amoureux de la musique, praticien ou auditeur exigeant, les textes présentés par Georges Goormaghtigh - lui-même joueur et enseignant de qin - possèdent une vertu indéfinissale qui tient à un subtil équilibre entre l'expérience intérieure du musicien et l'accord qu'il renouvelle sans cesse avec son instrument. On pourrait d'ailleurs étendre l'apport précieux de ces traités à l'art en général, tant les observations qu'ils véhiculent s'ajustent aux conditions préalables nécessaires à l'exercice de toute discipline artistique considérée comme un lien entre le visible et l'invisible.

3 Ji Kang, poète, philisophe et musicien chinois était l'un des fameux « Sept sages de la forêt de bambou ", groupe de lettrés taoisants qui se réunissaient "pour converser, boire, composer des poèmes et jouer de la musique ». Il vécut de 223 à 262 , au temps de la brève dynastie des Wei (220-265). La hache du bourreau écourta une existence consacrée à l'essentiel. Il avait 39 ans. Ji Kang laissait derrière lui son Qinfu, tenu pour un des textes fondateurs de la littérature esthétique chinoise par une postérité éclairée.

4 La « Description poétique du qin », traduite pour la première fois en français par Georges Goormaghtigh, retrace en vingt étapes (stations, sommes-nous tenté d'écrire) la conception et la création du qin - attribuée aux empereurs mythiques $\mathrm{Fu} \mathrm{Xi}$, le créateur des Trigrammes, et Shen Nong, le Divin Laboureur, grands ancêtres de la culture chinoise - par des sages fuyant le monde et inspirés par les paysages qu'ils contemplent.

5 "L'éveil à travers la contemplation du monde naturel est au cœur de la tradition du qin ». Le qindao, la "voie du qin», par-delà l'usage toujours plus affiné de l'instrument, le perfectionnement du doigté, pierre de touche de l'art du qin, s'affirme aussi comme le moyen d'une délivrance. C'est ainsi que « la musique du qin résume l'Harmonie du Milieu et unit tous les êtres ", selon le commentaire pertinent de Georges Goormaghtigh.

$6 \quad$ Nous serions tenté de citer de nombreux passages de cette remarquable traduction - bien que nous soyons incompétent pour juger de la qualité proprement linguistique du travail, ignorant tout des idéogrammes chinois - tant s'y fait oublier la volonté personnelle du traducteur. Nous ne sentons pas dans le texte de présence inopportune, mais plutôt la présence du passeur qu'est tout traducteur qui épouse le rythme inscrit dans la prose et les vers du poème. La subjectivité de l'homme du $\mathrm{XX}^{\mathrm{e}}$ siècle ne s'interpose pas entre le sens et la langue d'un écrit du III ${ }^{\mathrm{e}}$ siècle. A tout le moins est-elle contenue.

7 L'approche sensible et respectueuse - sans servilité ni obséquiosité - de Georges Goormaghtigh permet à la force d'évocation, à la richesse poétique, à la beauté simple serties dans le texte chinois d'aller leur chemin, telle une rivière sinuant entre les obstacles. Le traducteur s'est imposé le plus difficile : accompagner l'onde libérée par Ji Kang et veiller à ce que nul écueil n'interrompe sa course. C'est un des secrets de la traduction poétique. Etre vigilant, s'imprégner profondément des impressions provoquées par le texte original, laisser les résonances cristallines de la parole poétique se propager dans tout l'être (nous faisons allusion notamment au passage englobant les parties XVI à XVIII : « Mais qui n'est pas ouvert et détaché ne trouve pas sa joie dans le qin »), puis les restituer au plus près de ce que l'on a reçu.

8 Il y faut toute la science du traducteur; mais cela seul ne suffit pas: il faut encore accueillir le sens occulté, l'intention invisible, ce que les mots sont incapables de rendre sinon par leur soumission à une volonté absente de toute logique littéraire. L'acte poétique, à l'instar de l'exercice du qin défendu par Ji Kang, engendre l'harmonie et apparent paradoxe - est engendré par elle. "La pratique du qin est d'ailleurs conçue comme une voie de réalisation pour celui qui en joue », relève avec justesse Georges 
Goormaghtigh. L'influence du Tao, prépondérante dans l'œuvre, se révèle au long des vingt chants de la «Description». Ce n'est pas le moindre mérite de Georges Goormaghtigh d'avoir libéré ce parfum sans odeur de l'urne funéraire du Qinfu.

9 La seconde partie de l'ouvrage comprend « Les vingt-quatre saveurs du qin », un traité dû à Xu Shangying, joueur de qin renommé en son temps et dont on sait peu de choses. Il serait né à la fin du XVI ${ }^{e}$ siècle (1582 ?-1657 ?) dans la province du Jiangsu. Le traité se compose de vingt-quatre textes en prose, d'inégale longueur, et éclairant les multiples aspects du qin, de l'harmonie à la rapidité. Vingt-quatre «saveurs" abordant simultanément les plans poétique, philosophique et technique de cet instrument-roi. L'influence de la nature, l'importance de l'énergie vitale, la dimension cosmologique du concept d'harmonie, la prise en compte finalement de tous ces éléments réunis autorise à écrire que la pratique du qin conduit à une expérience se situant au-delà de la musique ou d'une simple expression artistique; tout en demeurant par ailleurs un témoignage sur l'art du qin et sur l'esthétique musicale chinoise, de surcroît un des aboutissements les plus achevés de cette tradition.

On retrouve dans cette traduction (également inédite, excepté le chapitre II) la discrétion vigilante, l'élégance de la phrase et le souci du rythme, propres à la manière de Georges Goormaghtigh. Les vingt-quatre chapitres sont de petits joyaux littéraires tout empreints de simplicité et d'acuité poétique. Le Silence, la Lumière, la Pureté, la Fermeté, la Finesse, la Fluidité, la Vigueur, la Légèreté ou la Lenteur y sont scrutés avec un regard éminemment calme et profond. L'empathie entre le musicien chinois et le musicien genevois, à presque quatre siècles de distance, donne à la traduction des «Vingt-quatre saveurs du qin" cette fluidité admirable et bon nombre des qualités précisément détaillées par Xu Shangying dans son ouvrage.

11 La poésie supportant mal l'entourage d'un appareil critique, nous avons apprécié la lisibilité des textes sans surcharge inutile. Mais que les amateurs de notes ne s'alarment pas, ils les retrouveront, nettes et érudites, entre la traduction et l'original. Avec tout ce qu'il faut d'introductions, de présentations, de bibliographies, d'index et autres annexes. Pour notre part, nous préférons aux notes utiles à l'érudit, celles dont le chapitre XXI des "Vingt-quatre saveurs ", intitulé "La légèreté », dit: «L'Harmonie du Milieu est dans une légèreté sans inconsistance et dans un poids sans violence. Ainsi, la légèreté et le poids ne sont-ils que les métamorphoses de l'Harmonie du Milieu et ce qui les rend tels, ce sont les notes véritables nées de l'Harmonie du Milieu ». 